\title{
Chronische myeloische Leukämie
}

\section{5-Jahres-Daten der DASISION-Studie: Frühe, tiefe und lang anhaltende Remission unter Dasatinib}

Tyrosinkinasehemmer (TKI) sind heute etablierter Bestandteil in der Behandlung der chronischen myeloischen Leukämie (CML). Insbesondere die TKI der 2. Generation wie Dasatinib $\left(\right.$ Sprycel $\left.^{\circledR}\right)$ führen in der Regel zu einem frühen und tiefen molekularen Ansprechen. Damit lässt sich, so die Daten der finalen 5-Jahres-Auswertung der DASISION-Studie, eine lang anhaltende Remission erreichen [1].

Mit Einführung von Tyrosinkinase-Inhibitoren (TKI) der 2. Generation wie Dasatinib konnte die Effektivität im Vergleich zum Erstgenerations-TKI Imatinib verbessert werden. Zudem ist Dasatinib auch bei Imatinib-Resistenz oder -Intoleranz einsetzbar [2].

Dasatinib hemmt als Multikinase-Inhibitor die Aktivität der BCR-ABL-Kinase, die für die onkogene Transformation der betroffenen hämatopoetischen Stammzelle verantwortlich ist. Darüber hinaus werden Kinasen der Src-Familie zusammen mit einer Reihe anderer ausgesuchter onkogener Kinasen wie c-Kit, Ephrin-Rezeptoren (Ephs) und der PDGF $\beta$-Rezeptor inhibiert. Dasatinib bindet an die inaktive und aktive Konformation des BCR-ABL-Enzyms, die mittlere terminale Halbwertszeit beträgt ca. 5 bis 6 Stunden. Dasatinib ist angezeigt für die Behandlung erwachsener Patienten mit neu diagnostizierter Philadelphia-Chromosom-positiver ( $\mathrm{Ph}+$ ) $\mathrm{CML}$ in der chronischen Phase und CML in der chronischen oder akzelerierten Phase oder in der Blastenkrise mit Resistenz oder Intoleranz gegenüber einer vorherigen Behandlung einschließlich Imatinibmesilat [2].

\section{DASISION-Studie:}

\section{Schnelles und tiefes Ansprechen} in der Erstlinie

Dass ein frühes molekulares Ansprechen auf einen TKI für den Langzeitverlauf der CML entscheidend ist, haben verschiedene Studien belegt [3-5]. Das European LeukemiaNET definierte für optimales Ansprechen BCR-ABL ${ }^{\text {IS }}$ $\leq 10 \%$ nach 3 Monaten als Meilenstein [6]. Patienten, die diesen BCR-ABL-Wert nach internationalem Standard erreichen [6], weisen die höchste Wahrscheinlichkeit für einen günstigen Langzeitverlauf auf.

Wie die Daten des finalen Follow-ups der zulassungsrelevanten Phase-III-Studie DASISION (DASatinib versus Imatinib In treatment-Naive CML patients) über 5 Jahre zeigen, erreichen signifikant mehr Studienteilnehmer unter Dasatinib in der Erstlinientherapie das Kriterium BCR-ABL ${ }^{\mathrm{IS}} \leq 10 \%$ nach 3 Monaten im Vergleich zu Imatinib ( $84 \%$ vs. $64 \%$; $p=0,0001$ ) $[1,7]$. Damit haben potenziell mehr Patienten unter dem TKI der 2. Generation die Chance, ein gutes Langzeitoutcome zu erreichen.

Die DASISION-Studie untersuchte die Wirksamkeit und Sicherheit sowie Prognose einer Firstline-Therapie mit Dasatinib $(1 \times$ täglich $100 \mathrm{mg} ; \mathrm{n}=259)$ im Vergleich zu Imatinib ( $1 \times$ täglich $400 \mathrm{mg} ; \mathrm{n}=260)$ bei erwachsenen Patienten mit neu diagnostizierter CML in der chronischen Phase (CP-CML). Das mediane Alter der Patienten betrug 46 Jahre in der Dasatinib- und 49 Jahre in der ImatinibGruppe, 8\% bzw. 9\% der Patienten waren 65 Jahre oder älter [7].

In der 12-Monats-Analyse erwies sich Dasatinib bezüglich des kompletten zytogenetischen Ansprechens (CCyR, primärer Endpunkt) versus Imatinib als signifikant überlegen ( $77 \%$ vs. $66 \% ; p=0,007)$. Das gilt auch für den sekundären Parameter gutes molekulares Ansprechen (major molecular remission, MMR) (46\% vs. $28 \%, \mathrm{p}<0,0001)$. Sowohl CCyR als auch MMR wurden zudem unter dem TKI der 2. Generation signifikant schneller erreicht (jeweils $\mathrm{p}<0,0001)$ [8].

\section{5-Jahres-Daten: \\ Überlegene Effektivität bei guter Verträglichkeit}

Mit den 24-Monats-Daten [8] und der vor Kurzem publizierten finalen Auswertung nach
5 Jahren Follow-up [1] der DASISION-Studie konnte die gute Wirksamkeit von Dasatinib bestätigt werden. So hatten nach 5 Jahren Erstlinientherapie signifikant mehr mit Dasatinibbehandelte Studienteilnehmer eine MMR erreicht $(76 \%$ vs. $64 \% ; p=0,0022)$ (Abb. 1 ). Bei $42 \%$ versus $33 \%$ der Patienten wurde eine BCR-ABL ${ }^{\text {IS }}$-Reduktion auf unter $0,0032 \%$ erzielt (Reduktion um 4,5-Log-Stufen, MR ${ }^{4,5}$; $\mathrm{p}=0,0251)$ [1].

Die geschätzte Gesamtüberlebensrate (OS) nach 5 Jahren lag für Dasatinib behandelte Patienten bei $91 \%$ sowie bei $90 \%$ im ImatinibArm (Hazard Ratio (HR) 1,01; 95\%-Konfidenzintervall (KI) 0,58-1,73) [1]. Auch nach Berücksichtigung der altersspezifischen Lebenserwartung zum Zeitpunkt der Diagnose errechnete sich für CP-CML-Patienten unter Therapie mit Dasatinib oder Imatinib eine Lebenserwartung, die nahezu mit der einer nicht an CML erkrankten Population vergleichbar war [1].

Numerisch starben in der Imatinib-Gruppe mehr Patienten $(\mathrm{n}=17)$ als in der DasatinibGruppe $(n=9)$, ohne dass sich beim 5-JahresÜberleben ein signifikanter Unterschied zeigte (HR 0,53; 95\%-KI 0,24-1,19; $\mathrm{p}=0,1192$ ). Die geschätzte Rate des progressionsfreien Überlebens (PFS) über 5 Jahre betrug 85\% unter Dasatinib und $86 \%$ im Imatinib-Arm (HR 1,06; 95\%-KI 0,68-1,66). Diese finalen Ergebnisse bestätigen nach Meinung der Autoren weiterhin, dass Dasatinib 100 mg 1-mal täglich eine verträgliche und wirksame Erstlinien-Therapie zur Langzeitbehandlung von CP-CML ist.[1].

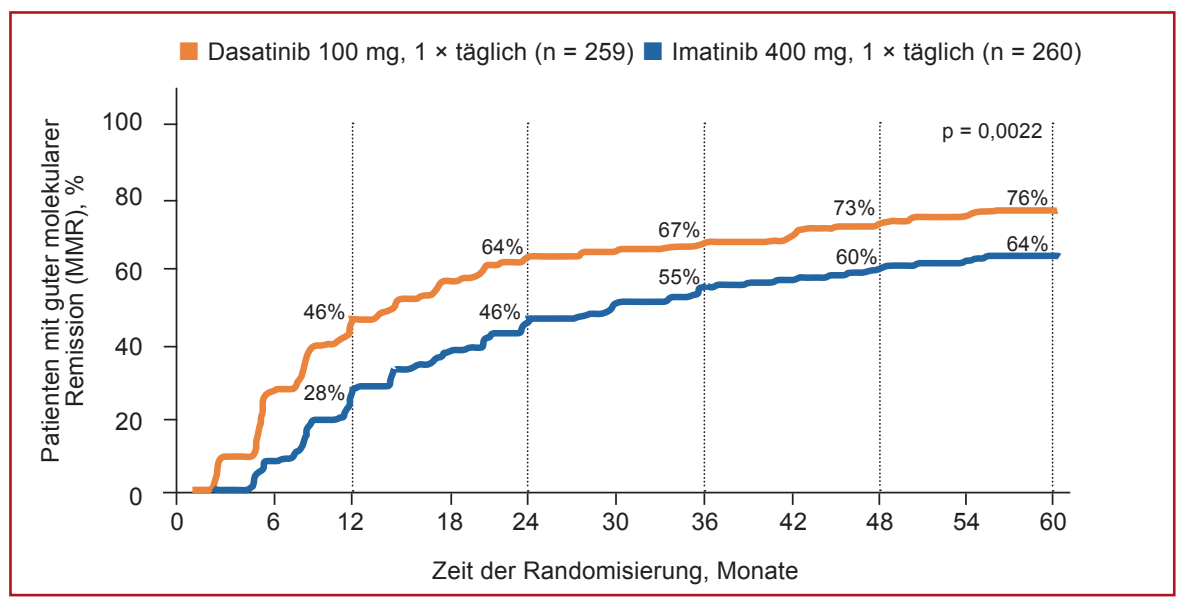

Abb. 1. Finale 5-Jahres-Auswertung der DASISION-Studie: Kumulative MMR-Ansprechraten über den Zeitverlauf unter Dasatinib und Imatinib; modifiziert nach [1]. 
Die Auswertung der Daten zur Verträglichkeit nach 5-jähriger Behandlung ergab für beide Medikationen keine neuen Sicherheitsbedenken. Mit Ausnahme von Pleuraergüssen wurden für Dasatinib seltener oder vergleichbar viele nicht hämatologische Nebenwirkungen berichtet wie für Imatinib. Nahezu alle Patienten (96\%) erreichten dabei trotz Entwicklung eines Pleuraergusses eine CCyR sowie $82 \%$ eine MMR. Nach Einschätzung der Autoren kann ein adäquates Management dazu beitragen, dass bei therapieinduzierten Pleuraergüssen die Therapie fortgesetzt wird. Nur 6\% der Studienteilnehmer brachen die Behandlung infolge dieser unerwünschten Wirkung ab [1].

\section{Gute Langzeitwirksamkeit unab- hängig vom Euro-Risikoscore}

Auf der Jahrestagung der Europäischen Gesellschaft für Hämatologie (EHA) wurde eine weitere Post-hoc-Analyse der DASISIONStudie vorgestellt. Die Auswertung zeigte, dass mehr Patienten unter Dasatinib als unter Imatinib ein frühes und tiefes optimales Ansprechen $\left(\mathrm{BCR}-\mathrm{ABL}^{\mathrm{IS}} \leq 10 \%\right)$ nach 3 Monaten erreichten. Damit war für diese Patienten über alle Euro-Risikoscores hinweg ein klinischer Langzeitnutzen unter dem ZweitgenerationsTKI verbunden [3]. Die Post-hoc-Analyse untersuchte den Einfluss des Euro-Risikoscores auf das Erreichen eines BCR-ABL ${ }^{\text {IS }}$ Werts $\leq 10 \%$ nach 3 und 6 Monaten sowie auf das Langzeitoutcome (PFS, OS, MMR, $\mathrm{MR}^{4,5}$ ) der Studienteilnehmer. Der Euro-Score berücksichtigt Parameter wie Alter, Milzgröße, Thrombozyten, Blastenanteil sowie Zahl der Eosinophilen und Basophilen [3].

Insgesamt wurden 474 der 519 Teilnehmer der DASISION-Studie für diese Auswertung eingeschlossen $(n=235$ im Dasatinib- und $n=239$ im Imatinib-Arm). Ein Euro-Score $\leq 780$ galt als Niedrigrisiko, zwischen 780 und 1480 als

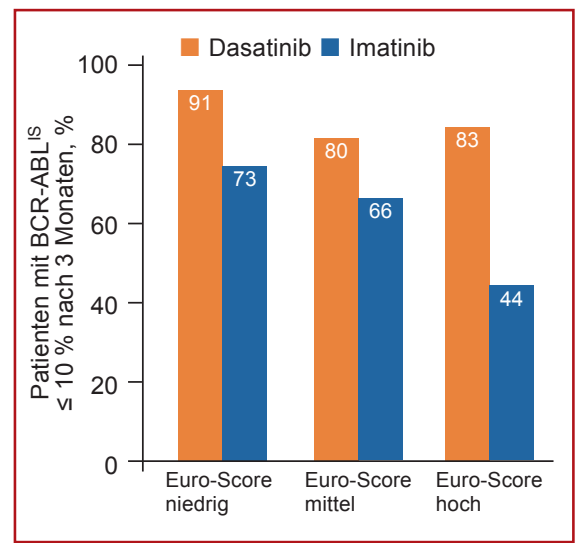

Abb. 2. Post-hoc-Analyse der DASISION-Studie: BCR-ABL ${ }^{\text {IS }}$-Werte nach 3 Monaten unter Dasatinib und Imatinib, stratifiziert nach dem Euro-Risikoscore; modifiziert nach [3]. intermediäres Risiko und > 1480 als Hochrisiko. $\mathrm{Zu}$ Studienbeginn wurden die meisten Patienten beider Medikationen dem intermediären Euro-Risikoscore zugeordnet ( $48 \%$ bzw. 47\%), gefolgt von Niedrigrisiko- (jeweils 33\%) und Hochrisikopatienten (jeweils 19\%) [3].

Im Vergleich mit den Patienten der ImatinibGruppe erreichten mehr Studienteilnehmer unter Dasatinib nach 3 Monaten einen BCR$\mathrm{ABL}^{\mathrm{IS}}$-Wert $\leq 10 \%$, unabhängig vom EuroRisikoscore (niedriges Risiko: 91\% vs. 73\%; mittleres Risiko: $80 \%$ vs. $66 \%$; hohes Risiko: $83 \%$ vs. $44 \%$ ) (Abb. 2). Der größte Unterschied zeigte sich in der Hochrisikogruppe: Hier erzielten mit Dasatinib 83\% der Patienten den BCR-ABL-Meilenstein nach 3 Monaten, mit Imatinib waren es $44 \%$. Nach 6 Monaten erreichten rund doppelt so viele Patienten einen BCR-ABL ${ }^{\mathrm{IS}}$-Wert $\leq 1 \%$ wie unter Imatinib (60\% vs. $29 \%$ ) [3]

Der Anteil der Patienten, der nach 3 Monaten einen noch tieferen BCR-ABL ${ }^{\mathrm{IS}}$-Wert $\leq 1 \%$ erzielen konnte, war unter der Dasatinib-Gabe in jeder Risikogruppe höher als unter Imatinib. Nach 6 Monaten erreichte lediglich ein Patient aus der Dasatinib-Gruppe (Niedrigrisikogruppe) diesen Wert nicht [3].

Die Behandlung mit Dasatinib verbesserte bei Teilnehmern mit einem frühzeitigen Erreichen des BCR-ABL ${ }^{\text {IS }}$-Werts $\leq 10 \%$ nach 3 und 6 Monaten in allen Euro-Risikogruppen das 5-Jahres-Langzeitoutcome (PFS, OS, transformationsfreies Überleben (TFS)). Alle anderen ausgewerteten Baseline-Parameter hatten keinen Einfluss auf das Überleben [3].

Die Mehrzahl der Patienten unter Dasatinib oder Imatinib mit einer $\mathrm{MR}^{4}$ konnten die tiefe molekulare Remission unabhängig von der Risikogruppe über mehr als 2 Jahre beibehalten, mit Ausnahme der Hochrisikogruppe im Imatinib-Arm. Studienteilnehmer mit intermediärem oder hohem Risiko im DasatinibArm wiesen im Vergleich mit Imatinib eine höhere Wahrscheinlichkeit für eine tiefe molekulare Remission in diesem Zeitraum auf [3]. Im Ergebnis, so die Autoren, bestätigen die Daten den Einsatz von Dasatinib bei neu diagnostizierten CP-CML-Patienten aller Risikogruppen, da sich mit dem TKI der 2. Generation in der Regel ein frühes und tiefes molekulares Ansprechen aufrechterhalten lässt [3].

\section{Erste Ergebnisse mit Dasatinib und pegyliertem Interferon}

Inwieweit sich mit der Kombination Dasatinib und pegyliertem Interferon (Peg-IFN) alpha $2 \mathrm{~b}$ ein additiver oder synergistischer Effekt bei CML-Patienten erzielen lässt, wurde in der norwegischen Phase-II-Studie NCT01725204 (NordCML007) untersucht [9].
39 neu diagnostizierte CP-CML-Patienten (77\% Männer, mittleres Alter 48 Jahre) erhielten $1 \times$ täglich $100 \mathrm{mg}$ Dasatinib über $3 \mathrm{Mo}$ nate als Monotherapie, anschließend wurde Peg-IFN subkutan in einer initialen Dosis von $15 \mu \mathrm{g} /$ Woche dazugegeben. Bei guter Verträglichkeit wurde nach 6 Monaten die Dosis auf $25 \mu \mathrm{g} /$ Woche bis Ende des Monats 15 über einen Gesamt-Peg-IFN-Behandlungszeitraum von einem Jahr erhöht. Als primärer Endpunkt galten frühe Sicherheitsdaten sowie die MMRRaten nach 12 Monaten [9].

Die Kombination wurde allgemein gut vertragen und das Nebenwirkungsprofil war handhabbar. 84\% der Studienteilnehmer verblieben auf der Peg-IFN-Dosis nach 12 Monaten und 91\% bzw. 73\% der geplanten Dasatinib- bzw. Peg-IFN-Dosen konnten verabreicht werden. Bei 1 bzw. 3 Patienten traten während des 1 . bzw. 2. Behandlungsjahres Pleuraergüsse auf [9].

Nach Hinzugabe von Peg-IFN ließ sich ein deutlicher Anstieg der Ansprechraten beobachten: So erzielten $10 \%, 57 \%, 84 \%$ und $89 \%$ der Patienten nach Monat 3, 6, 12 und 18 eine MMR. Nach einem Jahr erreichten $46 \%$ der Studienteilnehmer eine $\mathrm{MR}^{4}$ und 27\% eine $\mathrm{MR}^{4,5}$; es trat kein Progress zur akzelerierten Phase auf [9].

Der Frage nach dem bisher noch nicht geklärten Mechanismus für die Wirkverstärkung durch Peg-IFN wird derzeit in verschiedenen Untersuchungen nachgegangen [9].

Dr. Katrina Recker, Hamburg

\section{Literatur}

1 Cortes JE et al.: J Clin Oncol 2016;34:2333-2340.

2 Fachinformation Sprycel ${ }^{\circledR}$ Filmtabletten, Stand Juli 2016.

3 Stegelmann F et al.: Poster Presentation, Chronic Myeloid Leukemia - Clinical 2; in 21. Jahrestagung der European Haematology Association (EHA), Kopenhagen, 2016, abstr P617.

4 Cortes JE: J Clin Oncol 2012;30:223-224.

5 Hughes TP et al.: Blood 2014;123:1353-1360.

6 Baccarani M et al.: Blood 2013;122:872-884.

Kantarjian H et al.: N Engl J Med 2010;362: 2260-2270.

8 Kantarjian H et al.: Blood 2012;119:1123-1129.

9 Hjorth-Hansen $\mathrm{H}$ et al.: Leukemia 2016, in press. doi 10.1038/leu.2016.121.

\begin{tabular}{l} 
Impressum \\
\hline Chronische myeloische Leukämie \\
5-Jahres-Daten der DASISION-Studie: \\
Frühe, tiefe und lang anhaltende \\
Remission unter Dasatinib \\
PharmaForum in \\
ONcoLOGY RESEARCH AND TREATMENT 39 | 10 | 16 \\
(c) 2016 by S. Karger Verlag für Medizin \\
und Naturwissenschaften GmbH \\
Wilhelmstraße 20A \\
79098 Freiburg, Deutschland \\
\hline Mit freundlicher Unterstützung durch \\
Bristol-Myers Squibb GmbH \& Co. KGaA \\
Verlag, Herausgeber, Redaktion und Verlagsgeschäftsührung \\
übernehmen keine Verantwortung für den Inhalt dieser Rubrik.
\end{tabular}

